\title{
'Candidatus Phytoplasma', a taxon for the wall-less, non-helical prokaryotes that colonize plant phloem and insects
}

\author{
The IRPCM Phytoplasma/Spiroplasma Working Team - Phytoplasma \\ taxonomy group
}

Correspondence

G. Firrao

firrao@uniud.it
The trivial name 'phytoplasma' has been adopted to collectively name wall-less, non-helical prokaryotes that colonize plant phloem and insects, which were formerly known as mycoplasma-like organisms. Although phytoplasmas have not yet been cultivated in vitro, phylogenetic analyses based on various conserved genes have shown that they represent a distinct, monophyletic clade within the class Mollicutes. It is proposed here to accommodate phytoplasmas within the novel genus 'Candidatus (Ca.) Phytoplasma'. Given the diversity within 'Ca. Phytoplasma', several subtaxa are needed to accommodate organisms that share $<97.5 \%$ similarity among their $16 \mathrm{~S}$ rRNA gene sequences. This report describes the properties of ' $\mathrm{C} a$. Phytoplasma', a taxon that includes the species 'Ca. Phytoplasma aurantifolia' (the prokaryote associated with witches'-broom disease of small-fruited acid lime), 'Ca. Phytoplasma australiense' (associated with Australian grapevine yellows), 'Ca. Phytoplasma fraxini' (associated with ash yellows), 'Ca. Phytoplasma japonicum' (associated with Japanese hydrangea phyllody), 'Ca. Phytoplasma brasiliense' (associated with hibiscus witches'-broom in Brazil), 'Ca. Phytoplasma castaneae' (associated with chestnut witches'-broom in Korea), 'Ca. Phytoplasma asteris' (associated with aster yellows), 'Ca. Phytoplasma mali' (associated with apple proliferation), 'Ca. Phytoplasma phoenicium' (associated with almond lethal disease), 'Ca. Phytoplasma trifolii' (associated with clover proliferation), 'Ca. Phytoplasma cynodontis' (associated with Bermuda grass white leaf), 'Ca. Phytoplasma ziziphi' (associated with jujube witches'-broom), 'Ca. Phytoplasma oryzae' (associated with rice yellow dwarf) and six species-level taxa for which the Candidatus species designation has not yet been formally proposed (for the phytoplasmas associated with X-disease of peach, grapevine flavescence dorée, Central American coconut lethal yellows, Tanzanian lethal decline of coconut, Nigerian lethal decline of coconut and loofah witches'-broom, respectively). Additional species are needed to accommodate organisms that, despite their $16 \mathrm{~S}$ rRNA gene sequence being $>97.5 \%$ similar to those of other 'Ca. Phytoplasma' species, are characterized by distinctive biological, phytopathological and genetic properties. These include 'Ca. Phytoplasma pyri' (associated with pear decline), 'Ca. Phytoplasma prunorum' (associated with European stone fruit yellows), 'Ca. Phytoplasma spartii' (associated with spartium witches'-broom), 'Ca. Phytoplasma rhamni' (associated with buckthorn witches'-broom), 'Ca. Phytoplasma allocasuarinae' (associated with allocasuarina yellows), 'Ca. Phytoplasma ulmi' (associated with elm yellows) and an additional taxon for the stolbur phytoplasma.

Conversely, some organisms, despite their $16 \mathrm{~S}$ rRNA gene sequence being $<97.5 \%$ similar to that of any other 'Ca. Phytoplasma' species, are not presently described as Candidatus species, due to their poor overall characterization.
Published online ahead of print on 13 February 2004 as DOI 10.1099/ ijs.0.02854-0.

Abbreviations: Ca., Candidatus; IRPCM, International Research Programme for Comparative Mycoplasmology.

This paper is dedicated to the memory of Monique Garnier-Semancik, fine scientist and friend.

\section{INTRODUCTION}

Evidence that some plant diseases were associated with phloem colonization by prokaryotes that morphologically resembled mycoplasmas was first presented by Doi et al. (1967). Since then, several hundred plant syndromes have been associated with and thought to be caused by so-called 
'mycoplasma-like organisms' [reviewed by McCoy et al. (1989) and Seemüller et al. (1998)]. Due to their inability to grow in vitro, they were poorly characterized until the advent of molecular biology. Lately, rRNA gene sequencing has provided evidence that the non-spiroplasma, wall-less prokaryotes that colonize plant phloem and insects constitute a large, monophyletic group within the class Mollicutes (Lim \& Sears, 1989; Kuske \& Kirkpatrick, 1992; Sears \& Kirkpatrick, 1994). Therefore, at the 9th Congress of the International Organization of Mycoplasmology in 1992, the Phytoplasma Working Team of the International Research Project for Comparative Mycoplasmology (IRPCM) adopted the trivial name 'phytoplasma' to identify prokaryotes that belong to this group and to represent its present composition (International Committee on Systematic Bacteriology Subcommittee on the Taxonomy of Mollicutes, 1993). Since then, several distinct taxa have been described as 'Candidatus (Ca.) Phytoplasma aurantifolia' (Zreik et al., 1995), 'Ca. Phytoplasma australiense' (Davis et al., 1997), 'Ca. Phytoplasma australasia' (White et al., 1998), 'Ca. Phytoplasma fraxini' (Griffiths et al., 1999), 'Ca. Phytoplasma japonicum' (Sawayanagi et al., 1999), 'Ca. Phytoplasma brasiliense' (Montano et al., 2001), 'Ca. Phytoplasma castaneae' (Jung et al., 2002), 'Ca. Phytoplasma phoenicium' (Verdin et al., 2003), 'Ca. Phytoplasma ziziphi' (Jung et al., 2003a), 'Ca. Phytoplasma oryzae' (Jung et al., 2003b) and 'Ca. Phytoplasma ulmi' (Lee et al., 2004b), but a general description of the comprehensive taxon ' $\mathrm{Ca}$. Phytoplasma' itself has not yet been provided.

According to a recently adopted taxonomic rule (Murray \& Schleifer, 1994; Murray \& Stackebrandt, 1995), the properties of uncultured organisms should be recorded by a Candidatus designation. The scope of the present paper is to provide a formal description of the genus ' $\mathrm{Ca}$. Phytoplasma' and to summarize its species composition.

\section{METHODS}

$16 S$ rRNA gene sequence analysis. All GenBank records containing sequences that were defined, or could be referred to, as phytoplasma $16 \mathrm{~S}$ rRNA genes were downloaded. Partial sequences that contained $<1200$ bp within the first 1400 positions at the $5^{\prime}$ end of the 16S rRNA gene were discarded. Seventy of the 194 selected sequences were aligned manually by using the sequence editor SEQPUP (Gilbert, 1996). Using the profile alignment option of the program CLUSTAL_X (Thompson et al., 1997), the remaining 124 sequences were added to the manual alignment. Distances between sequences were calculated by using the method of Jukes \& Cantor (1969) by the DNADIST program of the PHYLIP package (Felsenstein, 1995). The alignment was deposited in TreeBase (http://www.treebase. org/treebase/) under the accession no. S1048-1787. A dendrogram was constructed with the neighbour-joining method of Saitou \& Nei (1987) with the CLUSTAL_X program (Thompson et al., 1997) and was used to assist group definition. Sequence signatures were determined with ALISCAN (De Marta \& Firrao, 2003). A second alignment that contains only the sequences of reference strains for the ' $\mathrm{Ca}$. Phytoplasma' species recognized in this work is available from TreeBase under the accession no. S1048-1788.

\section{RESULTS AND DISCUSSION}

Evidence for an unambiguously identifiable taxon to encompass all plant-pathogenic, non-helical Mollicutes has been provided by several independent phylogenetic analyses (Lim \& Sears, 1989; Kuske \& Kirkpatrick, 1992; Namba et al., 1993b; Gundersen et al., 1994; Kirkpatrick et al., 1994; Sears \& Kirkpatrick, 1994; Seemüller et al., 1994, 1998, 2002; Smart et al., 1994; Toth et al., 1994; Tymon et al., 1998; Lee et al., 2000). Accordingly, phytoplasmas represent a clearly distinct, monophyletic cluster within the class Mollicutes.

\section{Description of 'Candidatus Phytoplasma'}

'Candidatus Phytoplasma' (from phytos, Greek for plant and plasma, Greek for thing moulded) [(Mollicutes) NC; NA; O; NAS (GenBank accession no. M30790); oligonucleotide sequence of unique region of the 16S rRNA gene is CAAGAYBATKATGTKTAGCYGGDCT; P (Plant, phloem; Insect, salivary gland); M].

Morphology. 'Ca. Phytoplasma' cells are surrounded by a single-unit membrane, lack rigid cell walls and are pleomorphic in shape (Doi et al., 1967). When observed by transmission electron microscopy, they appear as rounded to filamentous, pleomorphic bodies with a mean diameter of 200-800 nm [reviewed by Kirkpatrick (1992) and Lee et al. (2000)].

Habitat. Organisms that belong to the genus ' $\mathrm{Ca}$. Phytoplasma' inhabit the phloem sieve elements (and, more rarely, also parenchymal cells) of vascular plants and the gut, haemolymph, salivary gland and other organs of sap-sucking insects. In insect hosts, they may cause premature mortality. In plant hosts, they may cause complex syndromes with specific symptoms, such as virescence, phyllody, sterility of flowers, proliferation of auxiliary or axillary shoots, abnormal elongation of internodes and many other, often less specific symptoms [reviewed by McCoy et al. (1989), Kirkpatrick (1992) and Lee et al. (2000)].

Antibiotic sensitivity. Members of the genus ' $\mathrm{Ca}$. Phytoplasma' are sensitive to antibiotics of the tetracycline group, but not to penicillin (Ishiie et al., 1967).

Base composition of DNA. According to results from buoyant density centrifugation, the $\mathrm{G}+\mathrm{C}$ content of ' $\mathrm{Ca}$. Phytoplasma' DNA is $23-29$ mol\% (Kollar \& Seemüller, 1989; Sears et al., 1989).

Chromosome size. The size of ' $\mathrm{Ca}$. Phytoplasma' chromosomes ranges from $530 \mathrm{kbp}$ to $1350 \mathrm{bp}$, as estimated from mobility on PFGE (Neimark \& Kirkpatrick, 1993; Marcone et al., 1999).

Codon usage. Members of the genus 'Ca. Phytoplasma' use UGA as a stop codon and not as a tryptophan codon, 
as in several other mycoplasmas (Lim \& Sears, 1991; Toth et al., 1994).

Sterols in cellular membrane. ' $\mathrm{Ca}$. Phytoplasma' membranes are resistant to digitonin and sensitive to hypotonic salt solutions, and are therefore similar to those of non-sterol-requiring mollicutes (Lim et al., 1992).

rRNA. Specific nucleotide signatures that are characteristic of ' $\mathrm{Ca}$. Phytoplasma' are: A at position 242, T at position 286 and $\mathrm{T}$ at position 1247 (in the sequence with GenBank accession no. M30790). Oligonucleotides that have been shown to specifically target ' $\mathrm{Ca}$. Phytoplasma' 16S rRNA genes in PCRs have been published (Deng \& Hiruki, 1991; Ahrens \& Seemüller, 1992; Firrao et al., 1993; Lee et al., 1993b; Namba et al., 1993a; Schneider et al., 1993; Padovan et al., 1995; Gundersen \& Lee, 1996). All strains of ' $\mathrm{Ca}$. Phytoplasma' that have been investigated so far have two rRNA operons (Schneider \& Seemüller, 1994; Firrao et al., 1996b; Lauer \& Seemüller, 2000; Padovan et al., 2000; Marcone \& Seemüller, 2001) and, usually, a single tRNA ${ }^{\text {Ile }}$ in the spacer region between the 16S and 23S rRNA genes (Kuske \& Kirkpatrick, 1992; Smart et al., 1996). Heterogeneity of the two operons is apparent in some phytoplasmas (Lee et al., 1993b; Firrao et al., 1996a; Liefting et al., 1996; Davis \& Sinclair, 1998; Jomantiene et al., 2002).

\section{Species belonging to 'Ca. Phytoplasma'}

A similarity matrix that included all $16 \mathrm{~S}$ rRNA gene sequences of named phytoplasma strains that were deposited in public databases was constructed. Sequences were ordered in a dendrogram and groups of sequences that shared $97.5 \%$ or more similarity among themselves and $<97.5 \%$ similarity with any other sequence were identified. One sequence from each group was selected as representative and the corresponding strain is described as a subtaxon within the 'Ca. Phytoplasma' taxon. According to Stackebrandt \& Goebel (1994), 'at sequence homology values below about $97.5 \%$ [in the 16S rRNA gene], it is unlikely that two organisms have more than 60 to $70 \%$ DNA similarity and hence that they are related at the species level'.

A congruent amount of results has already been produced in recent years, showing that phytoplasma strains that have $<97.5 \%$ similarity in their $16 \mathrm{~S}$ rRNA gene sequence actually represent different organisms, on the basis of phylogenetic analysis based on 16S rRNA gene sequences (Namba et al., 1993b; Gundersen et al., 1994; Seemüller et al., 1994, 1998; Tymon et al., 1998; Lee et al., 2000), 16S23S rRNA gene spacer sequences (Kirkpatrick et al., 1994), 23S rRNA gene sequences (Guo et al., 1998), ribosomal protein gene sequences (Jomantiene et al., 1998; Lee et al., 1998), transcription factor Tu gene (Schneider et al., 1997a), restriction analysis of the same genes (Schneider et al., 1993, 1997a, b; Vibio et al., 1996; Lee et al., 1998), RFLP of total DNA with chromosomal probes (Lee \& Davis, 1988;
Bertaccini et al., 1990; Harrison et al., 1991, 1992; Hibben et al., 1991; Kuske et al., 1991; Lee et al., 1991, 1992a, b, 1993a; Daire et al., 1992; Davis et al., 1992a, b; Ahrens et al., 1993; Mäurer et al., 1993; Chen et al., 1994; Griffiths et al., 1994; Davis \& Sinclair, 1998) and biological properties, such as insect vector specificity (Tsai, 1979; Shiomi \& Sugiura, 1984; Kirkpatrick, 1992).

However, there is evidence that some groups that are defined at $97.5 \%$ or higher $16 \mathrm{~S}$ rRNA gene sequence similarity include phytoplasma strains with very different biological (i.e. insect vector), phytopathological (i.e. host plant specificity and symptomatology) and molecular (i.e. chromosome size) characteristics. These strains need to be distinguished taxonomically. It has been highlighted in the past that in several taxonomic groups, organisms with highly similar or even identical $16 \mathrm{~S}$ rRNA gene sequences may nevertheless share $<70 \%$ DNA similarity (as estimated by DNA-DNA reassociation studies) and therefore belong to different species (Fox et al., 1992; Stackebrandt \& Goebel, 1994; Stackebrandt et al., 2002; Botti \& Bertaccini, 2003). In the case of the phytoplasmas, there are also practical reasons of concern, as organisms that share high 16S rRNA gene sequence similarity may cause different plant diseases, subject to quarantine regulations. Therefore, in addition to the minimal set of species belonging to ' $\mathrm{Ca}$. Phytoplasma' that are defined by $97 \cdot 5 \% 16 \mathrm{~S}$ rRNA gene sequence similarity, more Candidatus species have been defined to distinguish between those organisms that proved to be significantly different on the basis of biological and genetic properties.

In order to prevent nomenclatural confusion that may arise from the description of poorly differentiated novel taxa, the Phytoplasma/Spiroplasma Working Team of the IRPCM (2000) suggested rules for the description of organisms as novel taxa within ' $\mathrm{Ca}$. Phytoplasma', as follows.

(a) The 'Ca. Phytoplasma' species description should refer to a single, unique $16 \mathrm{~S}$ rRNA gene sequence ( $>1200 \mathrm{bp}$ ). The strain from which this sequence was obtained should be named the 'reference strain' and not the 'type strain'. Strains in which even minimal differences in the 16S rRNA gene sequence from the reference strain are detected do not 'belong' to the Candidatus species, but are 'related' to it.

(b) In general, a strain can be described as a novel ' $\mathrm{Ca}$. Phytoplasma' species if its $16 \mathrm{~S}$ rRNA gene sequence has $<97.5 \%$ similarity to that of any previously described ' $\mathrm{Ca}$. Phytoplasma' species.

(c) There are, however, cases of phytoplasmas that share $>97.5 \%$ of their $16 \mathrm{~S}$ rRNA gene sequence, but clearly represent ecologically separated populations and, therefore, may deserve description as separate species. For such cases, description of two different species is recommended only when all three of the following conditions apply: 
Table 1. List of $16 \mathrm{~S}$ rRNA gene sequences of strains related to 'Ca. Phytoplasma' species

Refer to database records (accession numbers are given) for full references. The 16S rRNA group according to Lee et al. (2000) is given in parentheses in the first column.

\begin{tabular}{|c|c|c|c|c|}
\hline Phylogenetic group & $\begin{array}{c}\text { 'Candidatus Phytoplasma' } \\
\text { species }\end{array}$ & $\begin{array}{l}\text { Reference of the species } \\
\text { description paper }\end{array}$ & $\begin{array}{c}\text { GenBank } \\
\text { accession no. }\end{array}$ & Database entry description \\
\hline Aster yellows group (16SrI) & 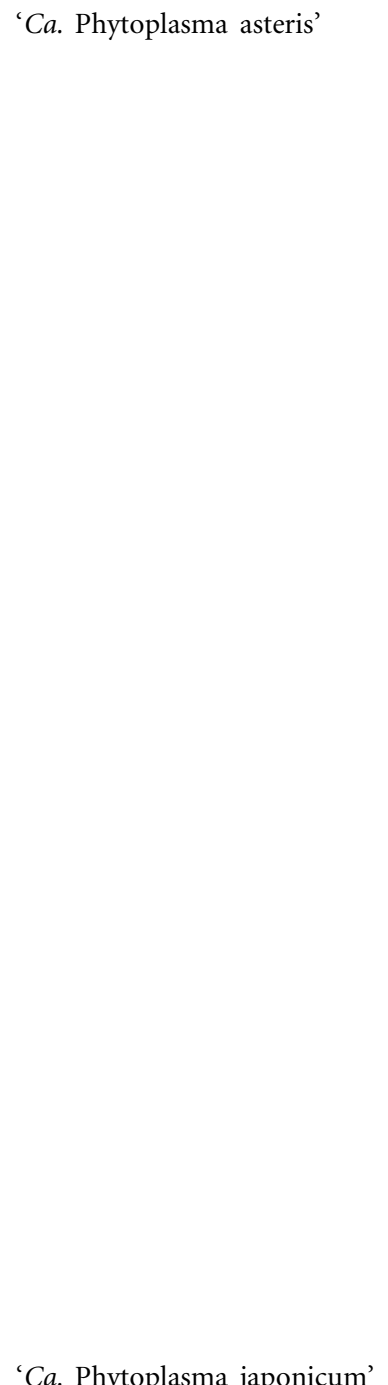 & Lee et al. (2004a) & $\begin{array}{l}\text { M30790 } \\
\text { M86340 } \\
\text { AF177384 } \\
\text { U96616 } \\
\text { L33760 } \\
\text { AF268403 } \\
\text { AF268404 } \\
\text { AF268405 } \\
\text { AF503568 } \\
\text { AY075038 } \\
\text { AF217247 } \\
\text { AF200431 } \\
\text { AF245439 } \\
\text { AF222064 } \\
\text { AF222063 } \\
\text { AF222065 } \\
\text { AF222066 } \\
\text { X68373 } \\
\text { U89378 } \\
\text { L33762 } \\
\text { D12569 } \\
\text { X68338 } \\
\text { AF268408 } \\
\text { AF268409 } \\
\text { AF322644 } \\
\text { AF291682 } \\
\text { AF335107 } \\
\text { AF453328 } \\
\text { AF279271 } \\
\text { AF356846 } \\
\text { AF322645 } \\
\text { L33767 } \\
\text { AF268407 } \\
\text { AF268406 } \\
\text { AB010425 }\end{array}$ & $\begin{array}{l}\text { Mycoplasma-like organism (strain OAY) } \\
\text { Mycoplasma-like sp. } \\
\text { Alfalfa stunt phytoplasma } \\
\text { Phytoplasma sp. STRAWB2 } \\
\text { Tomato big bud mycoplasma-like organism } \\
\text { Aster yellows phytoplasma A isolate 98UW159 } \\
\text { Aster yellows phytoplasma A isolate 98UW166A } \\
\text { Aster yellows phytoplasma O isolate 98UW166B } \\
\text { Aster yellows phytoplasma B } \\
\text { Mulberry dwarf phytoplasma } \\
\text { Potato purple top phytoplasma } \\
\text { Cirsium yellows phytoplasma } \\
\text { Aster yellows phytoplasma } \\
\text { Tomato big bud phytoplasma } \\
\text { Aster yellows phytoplasma } \\
\text { Clover phyllody phytoplasma strain CPh rrnA } \\
\text { Clover phyllody phytoplasma strain CPh rrnB } \\
\text { Mycoplasma-like organism (substrain AAY) } \\
\text { Phytoplasma sp. } \\
\text { Clover phyllody mycoplasma-like organism } \\
\text { Group I phytoplasma } \\
\text { Mycoplasma-like organism (substrain ACLR) } \\
\text { Aster yellows phytoplasma A isolate 99UW111 } \\
\text { Aster yellows phytoplasma B isolate 99UW108 } \\
\text { Aster yellows phytoplasma strain AY1 clone } 14 \text { A } \\
\text { Carrot proliferation phytoplasma } \\
\text { Rehmannia glutinosa var. purpurea phytoplasma } \\
\text { Phytoplasma sp. PY1 } \\
\text { Paulownia witches'-broom phytoplasma } \\
\text { Cactus phytoplasma 'Martinez-Soriano } 2001 \text { ' } \\
\text { Aster yellows phytoplasma strain AY1 clone } 10 \\
\text { Aster yellows mycoplasma-like organism } \\
\text { Aster yellows phytoplasma B isolate 99UW89 } \\
\text { Aster yellows phytoplasma A isolate 99UW93 } \\
\text { Phytoplasma sp. }\end{array}$ \\
\hline
\end{tabular}


Table 1. cont.

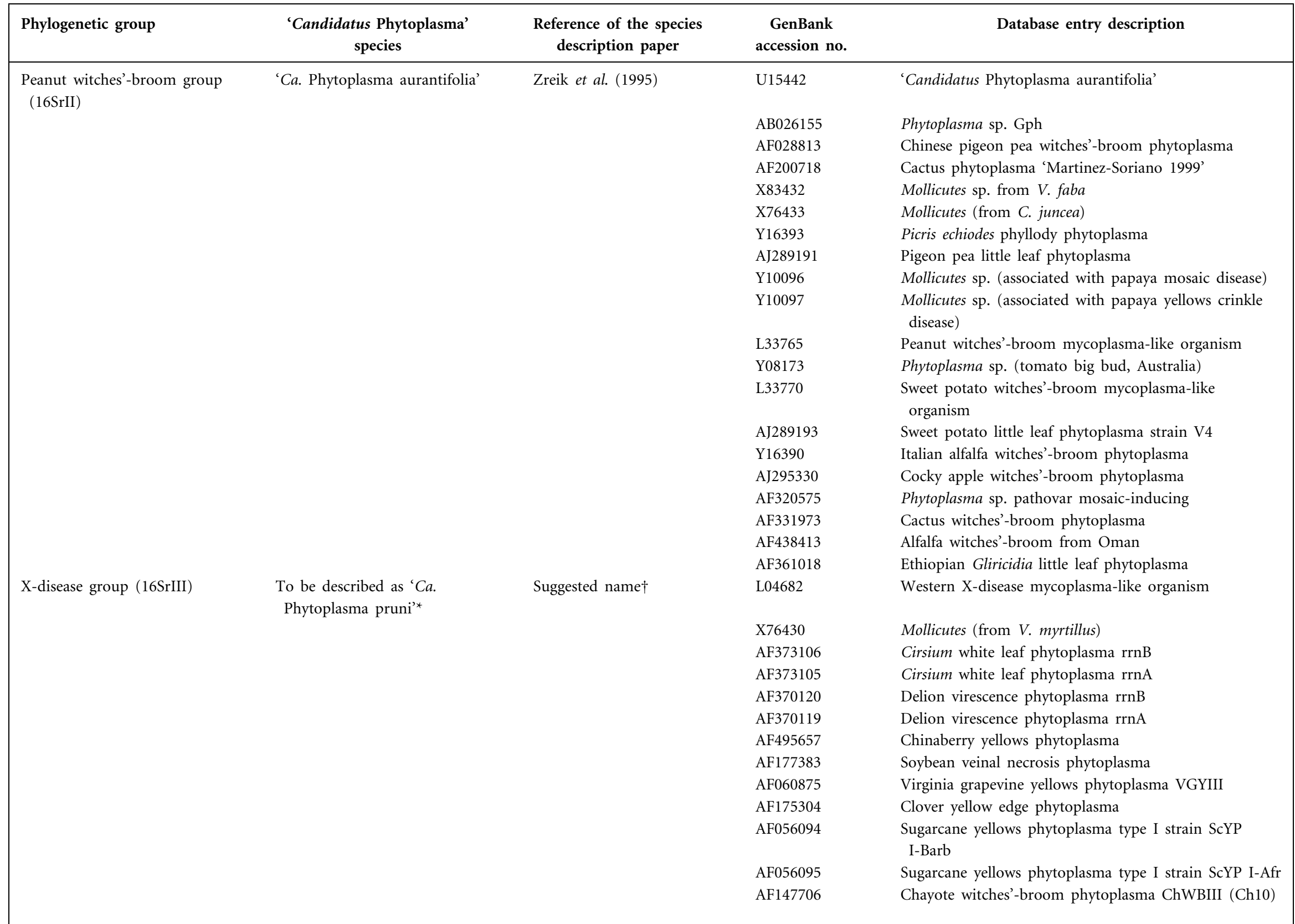


Table 1. cont.

\begin{tabular}{|c|c|c|c|c|}
\hline Phylogenetic group & $\begin{array}{c}\text { 'Candidatus Phytoplasma' } \\
\text { species }\end{array}$ & $\begin{array}{l}\text { Reference of the species } \\
\text { description paper }\end{array}$ & $\begin{array}{c}\text { GenBank } \\
\text { accession no. }\end{array}$ & Database entry description \\
\hline & & & AF147707 & Chayote witches'-broom phytoplasma ChWBIII (Mor5) \\
\hline & & & AF173558 & Clover yellow edge phytoplasma \\
\hline & & & AF236122 & Little peach phytoplasma \\
\hline & & & AF189288 & Clover yellow edge phytoplasma \\
\hline & & & AF236123 & Red suture phytoplasma \\
\hline & & & AF190223 & Poinsettia branch-inducing phytoplasma \\
\hline & & & AF190228 & Spiraea stunt phytoplasma \\
\hline & & & AF244363 & Black locust witches'-broom phytoplasma \\
\hline & & & L33733 & Canadian peach X mycoplasma-like organism \\
\hline & & & D12580 & Group II phytoplasma \\
\hline & & & X77482 & Mycoplasma-like organism (Italian clover phyllody) \\
\hline & & & L33766 & Clover yellow edge mycoplasma-like organism \\
\hline & & & AF302841 & $\begin{array}{l}\text { Black raspberry witches'-broom phytoplasma clone } \\
\text { BRWB7 }\end{array}$ \\
\hline & & & AF237615 & Carludovica palmata leaf yellowing phytoplasma \\
\hline & & & AF434989 & Texas Phoenix palm phytoplasma \\
\hline & & & U18753 & Yucatan coconut lethal decline phytoplasma \\
\hline & & & AF361020 & Florida Panus decline phytoplasma \\
\hline & $\begin{array}{l}\text { To be described as ' } \mathrm{Ca} \text {. } \\
\text { Phytoplasma cocostanzaniae'* }\end{array}$ & Suggested name $\ddagger$ & X80117 & Phytoplasma sp. strain LD \\
\hline & $\begin{array}{l}\text { To be described as 'Ca. } \\
\text { Phytoplasma cocosnigeriae'^ }\end{array}$ & Suggested name & Y14175 & Phytoplasma sp. strain LDN \\
\hline & & & Y13912 & Phytoplasma sp. strain LDG \\
\hline & 'Ca. Phytoplasma castaneae' & Jung et al. (2002) & AB054986 & ‘Candidatus Phytoplasma castaneae’ \\
\hline \multirow[t]{4}{*}{ Elm yellows group (16SrV) } & ‘Ca. Phytoplasma ziziphi’ & Jung et al. (2003a) & AY072722 & Ziziphus jujube witches'-broom phytoplasma \\
\hline & & & AF279272 & Ziziphus jujube witches'-broom phytoplasma \\
\hline & & & AF305240 & Ziziphus jujube witches'-broom phytoplasma \\
\hline & $\begin{array}{l}\text { To be described as 'Ca. } \\
\text { Phytoplasma vitis'^ }\end{array}$ & Suggested name $\dagger$ & AF176319 & Flavescence dorée phytoplasma \\
\hline
\end{tabular}


Table 1. cont.

\begin{tabular}{|c|c|c|c|c|}
\hline Phylogenetic group & $\begin{array}{c}\text { 'Candidatus Phytoplasma' } \\
\text { species }\end{array}$ & $\begin{array}{l}\text { Reference of the species } \\
\text { description paper }\end{array}$ & $\begin{array}{c}\text { GenBank } \\
\text { accession no. }\end{array}$ & Database entry description \\
\hline & & & AF122912 & Phytoplasma HD1 \\
\hline & & & AF122911 & Elm yellows phytoplasma strain WVEY \\
\hline & & & AF189214 & Elm yellows phytoplasma \\
\hline & & & L33763 & Elm yellows mycoplasma-like organism (rDNA) \\
\hline & & & Y16395 & Rubus stunt phytoplasma \\
\hline & & & X68376 & Mycoplasma-like organism (substrain ULW) \\
\hline & & & AF305198 & Virginia creeper phytoplasma \\
\hline & & & AY028789 & Alder yellows phytoplasma \\
\hline & & & Y16387 & Alder yellows phytoplasma \\
\hline \multirow[t]{10}{*}{ Clover proliferation group (16SrVI) } & 'Ca. Phytoplasma trifolii' & Hiruki \& Wang (2004) & AY390261 & 'Ca. Phytoplasma trifolii’ \\
\hline & & & L33761 & Clover proliferation mycoplasma-like organism (rDNA) \\
\hline & & & AF036354 & Fragaria multicipita phytoplasma \\
\hline & & & AF190224 & Fragaria multicipita phytoplasma $\mathrm{rrnA}$ \\
\hline & & & AF190225 & Fragaria multicipita phytoplasma rrnB \\
\hline & & & X83431 & Mollicutes sp. from S. melongena \\
\hline & & & AF228052 & Brinjal little leaf phytoplasma \\
\hline & & & AF228053 & Periwinkle little leaf phytoplasma \\
\hline & & & AF409069 & Clover proliferation phytoplasma strain EY-IL 2 \\
\hline & & & AF409070 & Clover proliferation phytoplasma strain EY-IL 1 \\
\hline \multirow[t]{7}{*}{ Ash yellows group (16SrVII) } & 'Ca. Phytoplasma fraxini' & Griffiths et al. (1999) & AF092209 & Ash yellows phytoplasma \\
\hline & & & AF105316 & Ash yellows phytoplasma strain AshY5 \\
\hline & & & AF105315 & Ash yellows phytoplasma strain AshY3 \\
\hline & & & AF105317 & Ash yellows phytoplasma strain LWB3 \\
\hline & & & AF189215 & Ash yellows phytoplasma \\
\hline & & & X68339 & Mycoplasma-like organism (substrain ASHY) \\
\hline & & & L33759 & Ash yellows mycoplasma-like organism (rDNA) \\
\hline \multirow[t]{4}{*}{$\begin{array}{l}\text { Loofah witches'-broom group } \\
\text { (16SrVIII) }\end{array}$} & $\begin{array}{l}\text { To be described as ' } \mathrm{Ca} \text {. } \\
\text { Phytoplasma luffae” }\end{array}$ & Suggested name $\dagger$ & AF086621 & Loofah witches'-broom phytoplasma \\
\hline & & & AF353090 & $\begin{array}{l}\text { Loofah witches'-broom phytoplasma str. LfWB clone1 } \\
\text { rrnB }\end{array}$ \\
\hline & & & AF248956 & Loofah witches'-broom phytoplasma \\
\hline & & & L33764 & Loofah witches'-broom mycoplasma-like organism \\
\hline \multirow{5}{*}{$\begin{array}{l}\text { Pigeon pea witches'-broom group } \\
\text { (16SrIX) }\end{array}$} & 'Ca. Phytoplasma phoenicium' & Verdin et al. (2003) & AF515636 & 'Candidatus Phytoplasma phoenicium’ \\
\hline & & & AF515637 & 'Candidatus Phytoplasma phoenicium' \\
\hline & & & AF248957 & Pigeon pea witches'-broom phytoplasma \\
\hline & & & AF455038 & Almond witches'-broom phytoplasma strain AlmWB3 \\
\hline & & & AF455041 & Almond witches'-broom phytoplasma strain AlmWB-N1 \\
\hline
\end{tabular}


Table 1. cont.

\begin{tabular}{|c|c|c|c|c|}
\hline Phylogenetic group & $\begin{array}{c}\text { 'Candidatus Phytoplasma' } \\
\text { species }\end{array}$ & $\begin{array}{l}\text { Reference of the species } \\
\text { description paper }\end{array}$ & $\begin{array}{c}\text { GenBank } \\
\text { accession no. }\end{array}$ & Database entry description \\
\hline & & & AF455040 & Almond witches'-broom phytoplasma strain AlmWB-P1 \\
\hline & & & AF390136 & Almond witches'- broom phytoplasma strain AlmWB1 \\
\hline & & & AF390137 & Almond witches'- broom phytoplasma strain AlmWB2 \\
\hline & & & AF455039 & Almond witches'-broom phytoplasma strain AlmWB4 \\
\hline & & & L33735 & Pigeon pea witches'-broom mycoplasma-like organism \\
\hline & & & Y18052 & Knautia arvensis phyllody phytoplasma $23 \mathrm{~S}$ \\
\hline & & & Y16389 & Picris echioides yellows phytoplasma \\
\hline & & & U18763 & Caribbean PPWB mycoplasma-like organism \\
\hline & & & AF361017 & Honduran Gliricidia little leaf phytoplasma \\
\hline & & & AF361019 & Florida Rhynchosia little leaf phytoplasma \\
\hline \multirow[t]{18}{*}{ Apple proliferation group (16SrX) } & 'Ca. Phytoplasma mali' & Seemüller \& Schneider (2004) & AJ542541 & Apple proliferation phytoplasma AP15 \\
\hline & & & AJ542542 & Apple proliferation phytoplasma AP1/93 \\
\hline & & & AF248958 & Apple proliferation phytoplasma \\
\hline & & & X68375 & Mycoplasma-like organism (substrain AT) \\
\hline & & & X72206 & Apple proliferation MLO \\
\hline & 'Ca. Phytoplasma pyri' & Seemüller \& Schneider (2004) & AJ542543 & Pear decline phytoplasma PD1 \\
\hline & & & X76425 & Mollicutes \\
\hline & & & Y16392 & Pear decline phytoplasma \\
\hline & & & Y16394 & Peach yellow leafroll phytoplasma \\
\hline & 'Ca. Phytoplasma prunorum’ & Seemüller \& Schneider (2004) & AJ542544 & European stone fruit yellows phytoplasma ESFY-G1 \\
\hline & & & AJ542545 & European stone fruit yellows phytoplasma ESFY-G2 \\
\hline & & & AY029540 & European stone fruit yellows phytoplasma \\
\hline & & & $\mathrm{X} 77372$ & Mycoplasma-like organism (plum leptonecrosis) \\
\hline & & & X68374 & Mycoplasma-like organism (substrain PPER) \\
\hline & & & Y11933 & Phytoplasma sp. \\
\hline & 'Ca. Phytoplasma spartii' & Marcone et al. (2004a) & X92869 & Phytoplasma sp. \\
\hline & 'Ca. Phytoplasma rhamni' & Marcone et al. (2004a) & $\mathrm{X} 76431$ & Mollicutes (from $R$. frangula) \\
\hline & 'Ca. Phytoplasma allocasuarinae' & Marcone et al. (2004a) & AY135523 & 'Allocasuarina muelleriana' phytoplasma \\
\hline Rice yellow dwarf group (16SrXI) & 'Ca. Phytoplasma oryzae' & Jung et al. (2003b) & D12581 & Group III phytoplasma \\
\hline \multirow[t]{8}{*}{ Stolbur group (16SrXII) } & 'Ca. Phytoplasma australiense' & Davis et al. (1997) & L76865 & Australian grapevine yellows phytoplasma \\
\hline & & & U43570 & Phormium yellow leaf phytoplasma rrnB \\
\hline & & & U43569 & Phormium yellow leaf phytoplasma rrnA \\
\hline & & & AJ243045 & Strawberry lethal yellows phytoplasma \\
\hline & & & AJ243044 & Strawberry green petal phytoplasma \\
\hline & $\begin{array}{l}\text { To be described as ' } \mathrm{Ca} \text {. } \\
\text { Phytoplasma solani'^ }\end{array}$ & Suggested name $\dagger$ & AF248959 & Stolbur phytoplasma \\
\hline & & & X76427 & Mollicutes (from C. anuum to C. roseus) \\
\hline & & & X76428 & Mollicutes (from V. vinifera) \\
\hline
\end{tabular}


Table 1. cont.

\begin{tabular}{|c|c|c|c|c|}
\hline Phylogenetic group & $\begin{array}{c}\text { 'Candidatus Phytoplasma' } \\
\text { species }\end{array}$ & $\begin{array}{l}\text { Reference of the species } \\
\text { description paper }\end{array}$ & $\begin{array}{c}\text { GenBank } \\
\text { accession no. }\end{array}$ & Database entry description \\
\hline BGWL group (16SrXIV) & 'Ca. Phytoplasma cynodontis' & Marcone et al. (2004b) & $\begin{array}{l}\text { AJ550984 } \\
\text { AJ550985 } \\
\text { AJ550986 } \\
\text { AF248961 } \\
\text { AF509321 }\end{array}$ & $\begin{array}{l}\text { Bermuda grass white leaf phytoplasma } \\
\text { Bermuda grass white leaf phytoplasma } \\
\text { Bermuda grass white leaf phytoplasma } \\
\text { Bermuda grass white leaf phytoplasma } \\
\text { Cynodon white leaf phytoplasma }\end{array}$ \\
\hline $\begin{array}{l}\text { 'Ca. Phytoplasma brasiliense' group ( } 16 \mathrm{SrXV}) \\
\text { Other phytoplasmas not related to the above } \\
\text { Candidatus species according to } 16 \mathrm{~S} \text { rRNA } \\
\text { gene sequences: }\end{array}$ & 'Ca. Phytoplasma brasiliense' & Montano et al. (2001) & AF147708 & Hibiscus witches'-broom phytoplasma strain HibWB26 \\
\hline $\begin{array}{l}\text { Mexican periwinkle virescence group } \\
\text { (16SrXIII) }\end{array}$ & No name suggested & & $\begin{array}{l}\text { AF248960 } \\
\text { U96614 }\end{array}$ & $\begin{array}{l}\text { Mexican periwinkle virescence phytoplasma } \\
\text { Phytoplasma sp. STRAWB1 }\end{array}$ \\
\hline Not assigned & No name suggested & & $\begin{array}{l}\text { AF495882 } \\
\text { AJ289195 } \\
\text { Y16391 } \\
\text { AJ310849 } \\
\text { Y17055 } \\
\text { AJ289192 } \\
\text { X76429 } \\
\text { X76432 } \\
\text { X83438 } \\
\text { AF509324 } \\
\text { AF509325 } \\
\text { Y15865 }\end{array}$ & $\begin{array}{l}\text { Chinaberry yellows phytoplasma strain CbY1 } \\
\text { Vigna little leaf phytoplasma } \\
\text { Bindweed yellows phytoplasma } \\
\text { Phytoplasma sp. PinP } \\
\text { Phytoplasma sp. (strain StLL) } \\
\text { Stylosanthes little leaf phytoplasma } \\
\text { Mollicutes (from C. roseus) } \\
\text { Mollicutes (from S. officinarum) } \\
\text { Mollicutes sp. } 16 \mathrm{~S} \text { rRNA gene and tRNA-Ile } \\
\text { Sorghum grassy shoot phytoplasma variant I } \\
\text { Sorghum grassy shoot phytoplasma variant II } \\
\text { Phytoplasma sp. (strain GaLL) }\end{array}$ \\
\hline
\end{tabular}

${ }^{*}$ According to Rule $28 \mathrm{~b}$ of the Bacteriological Code, this is an incidental citation and does not constitute prior citation.

$\dagger$ Name proposed by the IRPCM Phytoplasma Working Team at the X International Congress of the International Organization of Mycoplasmology, Bordeaux, 1994.

\$Name proposed by the IRPCM Phytoplasma/Spiroplasma Working Team at the XIV International Congress of the International Organization of Mycoplasmology, Vienna, 2002. 
(i) the two phytoplasmas are transmitted by different vectors;

(ii) the two phytoplasmas have a different natural plant host (or, at least, their behaviour is significantly different in the same plant host);

(iii) there is evidence of significant molecular diversity, achieved by either hybridization to cloned DNA probes, serological reaction or PCR-based assay.

(d) The rank of subspecies should not be used.

(e) The reference strain should be made available to the scientific community from the authors of the Candidatus species description paper and it should be deposited (unless in vitro micropropagation proves impossible) in the micropropagated collection of Dr Assunta Bertaccini, DiSTA, Patologia Vegetale, Università di Bologna, Italy.

(f) Manuscripts that describe a novel ' $C a$. Phytoplasma' species should preferably be submitted to the International Journal of Systematic and Evolutionary Microbiology (IJSEM).

(g) The abbreviation for Candidatus is Ca. (e.g. 'Ca. Phytoplasma japonicum' stands for 'Candidatus Phytoplasma japonicum').

It is recommended that future descriptions of ' $\mathrm{Ca}$. Phytoplasma' species follow the above rules. In order to provide a standard reference for the calculation of $16 \mathrm{~S}$ rRNA gene sequence similarities between potential novel isolates and currently described ' $\mathrm{C}$. Phytoplasma' species, a reference sequence alignment is available from TreeBase under accession no. S1048-1788 (see Methods). For direct comparison and reference, strains that have been used to define each ' $\mathrm{Ca}$. Phytoplasma' species can be obtained from the collection of A. Bertaccini (see address above). For phytoplasmas that cannot be maintained in in vitro propagation collections, reference DNA (total DNA extracted from an infected plant) should be obtained from the corresponding author of the species description papers.

Table 1 reports a list of taxa that belong to ' $\mathrm{Ca}$. Phytoplasma', identified as described above, and a list of $16 \mathrm{~S}$ rRNA gene sequences of related strains. The listed taxa either have already been described individually in past issues of IJSEM or are described in accompanying papers in this issue or in forthcoming papers; the reader is referred to the original papers for their detailed descriptions (Zreik et al., 1995; Davis et al., 1997; White et al., 1998; Griffiths et al., 1999; Sawayanagi et al., 1999; Montano et al., 2001; Jung et al., 2002, 2003a, b; Hiruki \& Wang, 2004; Lee et al., 2004a, b; Marcone et al., 2004a, b; Seemüller \& Schneider, 2004). The taxon ' $\mathrm{Ca}$. Phytoplasma australasia' is not retained, as its $16 \mathrm{~S}$ rRNA gene sequence is $99.5 \%$ similar to that of ' $C a$. Phytoplasma aurantifolia' and there is no evidence that conditions (i) and (iii) of rule (c) above are satisfied.

\section{ACKNOWLEDGEMENTS}

The IRPCM Phytoplasma/Spiroplasma Working Team - Phytoplasma taxonomy group consists of: G. Firrao (head), Università di Udine, Italy; M. Andersen, HortResearch, Auckland, New Zealand; A. Bertaccini, Alma Mater Studiorum University of Bologna, Bologna, Italy; E. Boudon, INRA, Dijon, France; J. M. Bové, Université de Bordeaux 2 and INRA, I.B.V.M., Villenave d'Ornon, France; X. Daire, INRA, Dijon, France; R. E. Davis, ARS-USDA, Beltsville, MD, USA; J. Fletcher, Oklahoma State University, Stillwater, OK, USA; M. Garnier, INRA, Villenave d'Ornon, France; K. S. Gibb, Charles Darwin University, Darwin, Australia; D. E. Gundersen-Rindal, ARS-USDA, Beltsville, MD, USA; N. Harrison, University of Florida, Fort Lauderdale, FL, USA; C. Hiruki, University of Alberta, Edmonton, Canada; B. C. Kirkpatrick, University of California, Davis, CA, USA; P. Jones, Rothamsted Research, Harpenden, UK; C. R. Kuske, Los Alamos National Laboratory, Los Alamos, NM, USA; I.-M. Lee, ARSUSDA, Beltsville, MD, USA; L. Liefting, HortResearch, Auckland, New Zealand; C. Marcone, Università degli Studi della Basilicata, Potenza, Italy; S. Namba, University of Tokyo, Tokyo, Japan; B. Schneider, Biologische Bundesanstalt, Dossenheim, Germany; B. B. Sears, Michigan State University, East Lansing, MI, USA; E. Seemüller, Biologische Bundesanstalt, Dossenheim, Germany; C. D. Smart, Cornell University, Geneva, NY, USA; C. Streten, Charles Darwin University, Darwin, Australia; and K. Wang, University of Alberta, Edmonton, Canada.

\section{REFERENCES}

Ahrens, U. \& Seemüller, E. (1992). Detection of DNA of plant pathogenic mycoplasmalike organisms by a polymerase chain reaction that amplifies a sequence of the 16S rRNA gene. Phytopathology 82, 828-832.

Ahrens, U., Lorenz, K.-H. \& Seemüller, E. (1993). Genetic diversity among mycoplasmalike organisms associated with stone fruit diseases. Mol Plant-Microbe Interact 6, 686-691.

Bertaccini, A., Davis, R. E. \& Lee, I.-M. (1990). Distinctions among mycoplasmalike organisms (MLOs) in Gladiolus, Ranunculus, Brassica and Hydrangea through detection with non-radioactive cloned DNA probes. Phytopathol Mediterr 29, 107-113.

Botti, S. \& Bertaccini, A. (2003). Variability and functional role of chromosomal sequences in 16SrI-B subgroup phytoplasmas including aster yellows and related strains. J Appl Microbiol 94, 103-110.

Chen, K. H., Credi, R., Loi, N., Maixner, M. \& Chen, T. A. (1994). Identification and grouping of mycoplasmalike organisms associated with grapevine yellows and clover phyllody diseases based on immunological and molecular analyses. Appl Environ Microbiol 60, 1905-1913.

Daire, X., Boudon-Padieu, E., Bervillé, A., Schneider, B. \& Caudwell, A. (1992). Cloned DNA probes for detection of grapevine flavescence dorée mycoplasma-like organism (MLO). Ann Appl Biol 121, 95-103.

Davis, R. E. \& Sinclair, W. A. (1998). Phytoplasma identity and disease etiology. Phytopathology 88, 1372-1376.

Davis, R. E., Sinclair, W. A., Lee, I.-M. \& Dally, E. L. (1992a). Cloned DNA probes specific for detection of a mycoplasmalike organism associated with ash yellows. Mol Plant-Microbe Interact 5, 163-169.

Davis, R. E., Dally, E. L., Bertaccini, A., Credi, R., Lee, I.-M., Osler, R., Carraro, L. \& Barba, M. (1992b). Cloned DNA probes for specific detection of Italian periwinkle virescence mycoplasma-like organism (MLO) and investigation of genetic relatedness with other MLOs. Phytopathol Mediterr 31, 5-12. 
Davis, R. E., Dally, E. L., Gundersen, D. E., Lee, I.-M. \& Habili, N. (1997). "Candidatus Phytoplasma australiense," a new phytoplasma taxon associated with Australian grapevine yellows. Int $J$ Syst Bacteriol 47, 262-269.

De Marta, P. \& Firrao, G. (2003). ALISCAN. Development release (http://www.biodiv.it/aliscan/).

Deng, S. \& Hiruki, C. (1991). Amplification of $16 \mathrm{~S}$ rRNA genes from culturable and nonculturable mollicutes. J Microbiol Methods 14, 53-61.

Doi, Y., Teranaka, M., Yora, K. \& Asuyama, H. (1967). Mycoplasma or PLT group-like microorganisms found in the phloem elements of plants infected with mulberry dwarf, potato witches' broom, aster yellows, or Paulownia witches' broom. Ann Phytopathol Soc Jpn 33, 259-266.

Felsenstein, F. (1995). PHYLIP (Phylogeny Inference Package) version 3.57c. Seattle: University of Washington.

Firrao, G., Gobbi, E. \& Locci, R. (1993). Use of polymerase chain reaction to produce oligonucleotide probes for mycoplasmalike organisms. Phytopathology 83, 602-607.

Firrao, G., Carraro, L., Gobbi, E. \& Locci, R. (1996a). Molecular characterization of a phytoplasma causing phyllody in clover and other herbaceous hosts in northern Italy. Eur J Plant Pathol 102, 817-822.

Firrao, G., Smart, C. D. \& Kirkpatrick, B. C. (1996b). Physical map of the western X-disease phytoplasma chromosome. J Bacteriol 178, 3985-3988.

Fox, G. E., Wisotzkey, J. D. \& Jurtshuk, P., Jr (1992). How close is close: $16 \mathrm{~S}$ rRNA sequence identity may not be sufficient to guarantee species identity. Int J Syst Bacteriol 42, 166-170.

Gilbert, D. G. (1996). SEQPUP. Development release (ftp://iubio. bio.indiana.edu/molbio/seqpup/).

Griffiths, H. M., Gundersen, D. E., Sinclair, W. A., Lee, I.-M. \& Davis, R. E. (1994). Mycoplasmalike organisms from milkweed, goldenrod, and spirea represent two new 16S rRNA subgroups and three new strain subclusters related to peach X-disease MLOs. Can J Plant Pathol 16, 255-260.

Griffiths, H. M., Sinclair, W. A., Smart, C. D. \& Davis, R. E. (1999). The phytoplasma associated with ash yellows and lilac witches'broom: 'Candidatus Phytoplasma fraxini'. Int J Syst Bacteriol 49, 1605-1614.

Gundersen, D. E. \& Lee, I.-M. (1996). Ultrasensitive detection of phytoplasmas by nested-PCR assays using two universal primer pairs. Phytopathol Mediterr 35, 144-151.

Gundersen, D. E., Lee, I.-M., Rehner, S. A., Davis, R. E. \& Kingsbury, D. T. (1994). Phylogeny of mycoplasmalike organisms (phytoplasmas): a basis for their classification. J Bacteriol 176, 5244-5254.

Guo, Y. H., Cheng, Z.-M. \& Walla, J. A. (1998). Amplification and RFLP analysis of $23 \mathrm{~S}$ ribosomal DNA from phytoplasmas. Phytopathology 88, S35.

Harrison, N. A., Tsai, J. H., Bourne, C. M. \& Richardson, P. A. (1991) Molecular cloning and detection of chromosomal and extrachromosomal DNA of mycoplasmalike organisms associated with witches'-broom disease of pigeon pea in Florida. Mol Plant-Microbe Interact 4, 300-307.

Harrison, N. A., Bourne, C. M., Cox, R. L., Tsai, J. H. \& Richardson, P. A. (1992). DNA probes for detection of mycoplasmalike organisms associated with lethal yellowing disease of palms in Florida. Phytopathology 82, 216-224.

Hibben, C. R., Sinclair, W. A., Davis, R. E. \& Alexander, J. H., III (1991). Relatedness of mycoplasmalike organisms associated with ash yellows and lilac witches'-broom. Plant Dis 75, 1227-1230.
Hiruki, C. \& Wang, K. (2004). Clover proliferation phytoplasma: 'Candidatus Phytoplasma trifolii'. Int J Syst Evol Microbiol 54, 1349-1353.

International Committee on Systematic Bacteriology Subcommittee on the Taxonomy of Mollicutes (1993). Minutes of the interim meetings, 1 and 2 August, 1992, Ames, Iowa. Int J Syst Bacteriol 43, 394-397.

Ishiie, T., Doi, Y., Yora, K. \& Asuyama, H. (1967). Suppressive effects of antibiotics of tetracycline group on symptom development of mulberry dwarf disease. Ann Phytopathol Soc Jpn 33, 267-275.

Jomantiene, R., Davis, R. E., Maas, J. \& Dally, E. L. (1998). Classification of new phytoplasmas associated with diseases of strawberry in Florida, based on analysis of 16S rRNA and ribosomal protein gene operon sequences. Int J Syst Bacteriol 48, 269-277.

Jomantiene, R., Davis, R. E., Valiunas, D., Alminaite, A. \& Staniulis, J. (2002). New group 16SrIII phytoplasma lineages in Lithuania exhibit rRNA interoperon sequence heterogeneity. Eur J Plant Pathol 108, 507-517.

Jukes, T. H. \& Cantor, C. R. (1969). Evolution of protein molecules. In Mammalian Protein Metabolism, pp. 21-132. Edited by H. N. Munro. New York: Academic Press.

Jung, H.-Y., Sawayanagi, T., Kakizawa, S. \& 7 other authors (2002). 'Candidatus Phytoplasma castaneae', a novel phytoplasma taxon associated with chestnut witches' broom disease. Int J Syst Evol Microbiol 52, 1543-1549.

Jung, H.-Y., Sawayanagi, T., Kakizawa, S. \& 7 other authors (2003a). 'Candidatus Phytoplasma ziziphi', a novel phytoplasma taxon associated with jujube witches'-broom disease. Int J Syst Evol Microbiol 53, 1037-1041.

Jung, H. Y., Sawayanagi, T., Wongkaew, P. \& 8 other authors (2003b). 'Candidatus Phytoplasma oryzae', a novel phytoplasma taxon associated with rice yellow dwarf disease. Int J Syst Evol Microbiol 53, 1925-1929.

Kirkpatrick, B. C. (1992). Mycoplasma-like organisms: plant and invertebrate pathogens. In The Prokaryotes, 2nd edn, pp. 4050-4067. Edited by A. Balows, H. G. Trüper, M. Dworkin, W. Harder \& K. H. Schleifer. New York: Springer.

Kirkpatrick, B. C., Smart, C. D., Gardner, S. \& 9 other authors (1994). Phylogenetic relationship of plant pathogenic MLOs established by $16 / 23 \mathrm{~S}$ rDNA spacer sequences. IOM Lett 3, 228-229.

Kollar, A. \& Seemüller, E. (1989). Base composition of the DNA of mycoplasmalike organisms associated with various plant diseases. J Phytopathol 127, 177-186.

Kuske, C. R. \& Kirkpatrick, B. C. (1992). Phylogenetic relationships between the western aster yellows mycoplasmalike organisms and other prokaryotes established by $16 \mathrm{~S}$ rRNA gene sequence. Int J Syst Bacteriol 42, 226-233.

Kuske, C. R., Kirkpatrick, B. C., Davis, M. J. \& Seemüller, E. (1991). DNA hybridization between western aster yellows mycoplasmalike organism plasmids and extrachromosomal DNA from other plant pathogenic mycoplasmalike organisms. Mol Plant-Microbe Interact 4, $75-80$.

Lauer, U. \& Seemüller, E. (2000). Physical map of the chromosome of the apple proliferation phytoplasma. J Bacteriol 182, 1415-1418.

Lee, I.-M. \& Davis, R. E. (1988). Detection and investigation of genetic relatedness among aster yellows and other mycoplasma-like organisms by using cloned DNA and RNA probes. Mol PlantMicrobe Interact 1, 303-310.

Lee, I.-M., Davis, R. E. \& Hiruki, C. (1991). Genetic interrelatedness among clover proliferation mycoplasmalike organisms (MLOs) and other MLOs investigated by nucleic acid hybridization and 
restriction fragment length polymorphism analyses. Appl Environ Microbiol 57, 3565-3569.

Lee, I.-M., Davis, R. E., Chen, T.-A., Chiykowski, L. N., Fletcher, J., Hiruki, C. \& Schaff, D. A. (1992a). A genotype-based system for identification and classification of mycoplasmalike organisms (MLOs) in the aster yellows MLO strain cluster. Phytopathology 82, 977-986.

Lee, I.-M., Gundersen, D. E., Davis, R. E. \& Chiykowski, L. N. (1992b). Identification and analysis of a genomic strain cluster of mycoplasmalike organisms associated with Canadian peach (eastern) $\mathrm{X}$ disease, western X disease, and clover yellow edge. J Bacteriol 174, 6694-6698.

Lee, I.-M., Davis, R. E., Sinclair, W. A., DeWitt, N. D. \& Conti, M. (1993a). Genetic relatedness of mycoplasmalike organisms detected in Ulmus spp. in the United States and Italy by means of DNA probes and polymerase chain reactions. Phytopathology 83, 829-833.

Lee, I.-M., Hammond, R. W., Davis, R. E. \& Gundersen, D. E. (1993b). Universal amplification and analysis of pathogen $16 \mathrm{~S} \mathrm{rDNA}$ for classification and identification of mycoplasmalike organisms. Phytopathology 83, 834-842.

Lee, I.-M., Gundersen-Rindal, D. E., Davis, R. E. \& Bartoszyk, I. M. (1998). Revised classification scheme of phytoplasmas based on RFLP analyses of $16 \mathrm{~S}$ rRNA and ribosomal protein gene sequences. Int J Syst Bacteriol 48, 1153-1169.

Lee, I.-M., Davis, R. E. \& Gundersen-Rindal, D. E. (2000). Phytoplasma: phytopathogenic mollicutes. Annu Rev Microbiol 54, 221-255.

Lee, I.-M., Gundersen-Rindal, D. E., Davis, R. E., Bottner, K. D., Marcone, C. \& Seemüller, E. (2004a). 'Candidatus Phytoplasma asteris', a novel phytoplasma taxon associated with aster yellows and related diseases. Int J Syst Evol Microbiol 54, 1037-1048.

Lee, I.-M., Martini, M., Marcone, C. \& Zhu, S. F. (2004b). Classification of phytoplasma strains in the elm yellows group $(16 \mathrm{SrV})$ and proposal of 'Candidatus Phytoplasma ulmi' for the phytoplasma associated with elm yellows. Int J Syst Evol Microbiol 54, 337-347.

Liefting, L. W., Andersen, M. T., Beever, R. E., Gardner, R. C. \& Foster, R. L. S. (1996). Sequence heterogeneity in the two $16 \mathrm{~S}$ rRNA genes of Phormium yellow leaf phytoplasma. Appl Environ Microbiol 62, 3133-3139.

Lim, P.-O. \& Sears, B. B. (1989). 16S rRNA sequence indicates that plant-pathogenic mycoplasmalike organisms are evolutionarily distinct from animal mycoplasmas. J Bacteriol 171, 5901-5906.

Lim, P.-O. \& Sears, B. B. (1991). DNA sequence of the ribosomal protein genes rp12 and rps19 from a plant-pathogenic mycoplasmalike organism. FEMS Microbiol Lett 84, 71-73.

Lim, P.-O., Sears, B. B. \& Klomparens, K. L. (1992). Membrane properties of a plant-pathogenic mycoplasmalike organism. J Bacteriol 174, 682-686.

Marcone, C. \& Seemüller, E. (2001). A chromosome map of the European stone fruit yellows phytoplasma. Microbiology 147, $1213-1221$

Marcone, C., Neimark, H., Ragozzino, A., Lauer, U. \& Seemüller, E. (1999). Chromosome sizes of phytoplasmas composing major phylogenetic groups and subgroups. Phytopathology 89, 805-810.

Marcone, C., Gibb, K. S., Streten, C. \& Schneider, B. (2004a), 'Candidatus Phytoplasma spartii', 'Candidatus Phytoplasma rhamni' and 'Candidatus Phytoplasma allocasuarinae', respectively associated with spartium witches'-broom, buckthorn witches'-broom and allocasuarina yellows diseases. Int J Syst Evol Microbiol 54, 1025-1029.

Marcone, C., Schneider, B. \& Seemüller, E. (2004b). 'Candidatus Phytoplasma cynodontis', the phytoplasma associated with Bermuda grass white leaf disease. Int J Syst Evol Microbiol 54, 1077-1082.
Mäurer, R., Seemüller, E. \& Sinclair, W. A. (1993). Genetic relatedness of mycoplasmalike organisms affecting elm, alder, and ash in Europe and North America. Phytopathology 83, 971-976.

McCoy, R. E., Caudwell, A., Chang, C. J. \& 16 other authors (1989). Plant diseases associated with mycoplasma-like organisms. In The Mycoplasmas, vol. 5, pp. 545-640. Edited by R. F. Whitcomb \& J. G. Tully. San Diego, CA: Academic Press.

Montano, H. G., Davis, R. E., Dally, E. L., Hogenhout, S., Pimentel, J. P. \& Brioso, P. S. T. (2001). 'Candidatus Phytoplasma brasiliense', a new phytoplasma taxon associated with hibiscus witches'-broom disease. Int J Syst Evol Microbiol 51, 1109-1118.

Murray, R. G. E. \& Schleifer, K. H. (1994). Taxonomic notes: a proposal for recording the properties of putative taxa of procaryotes. Int J Syst Bacteriol 44, 174-176.

Murray, R. G. E. \& Stackebrandt, E. (1995). Taxonomic note: implementation of the provisional status Candidatus for incompletely described procaryotes. Int J Syst Bacteriol 45, 186-187.

Namba, S., Kato, S., Iwanami, S., Oyaizu, H., Shiozawa, H. \& Tsuchizaki, T. (1993a). Detection and differentiation of plantpathogenic mycoplasmalike organisms using polymerase chain reaction. Phytopathology 83, 786-791.

Namba, S., Oyaizu, H., Kato, S., Iwanami, S. \& Tsuchizaki, T. (1993b). Phylogenetic diversity of phytopathogenic mycoplasmalike orgnisms. Int J Syst Bacteriol 43, 461-467.

Neimark, H. \& Kirkpatrick, B. C. (1993). Isolation and characterization of full-length chromosomes from non-culturable plant pathogenic mycoplasma-like organisms. Mol Microbiol 7, 21-28.

Padovan, A. C., Gibb, K. S., Bertaccini, A., Vibio, M., Bonfiglioli, R. E., Magarey, P. A. \& Sears, B. B. (1995). Molecular detection of the Australian grapevine yellows phytoplasma and comparison with grapevine yellows phytoplasmas from Italy. Aust J Grape Wine Res 1, 25-31.

Padovan, A. C., Firrao, G., Schneider, B. \& Gibb, K. S. (2000). Chromosome mapping of the sweet potato little leaf phytoplasma reveals genome heterogeneity within the phytoplasmas. Microbiology 146, 893-902.

Phytoplasma/Spiroplasma Working Team of the International Research Project for Comparative Mycoplasmology (2000). Phytoplasmas, spiroplasmas, mesoplasmas, and entomoplasmas working team. In International Research Programme on Comparative Mycoplasmology (IRPCM) of the International Organization for Mycoplasmology (IOM) - Report of Consultations, Fukuoka, Japan, July 2000 (http://mycoplasmas.vm.iastate.edu/IOM/).

Saitou, N. \& Nei, M. (1987). The neighbor-joining method: a new method for reconstructing phylogenetic trees. Mol Biol Evol 4, 406-425.

Sawayanagi, T., Horikoshi, N., Kanehira, T., Shinohara, M., Bertaccini, A., Cousin M.-T., Hiruki, C. \& Namba, S. (1999). 'Candidatus Phytoplasma japonicum', a new phytoplasma taxon associated with Japanese Hydrangea phyllody. Int J Syst Bacteriol 49, 1275-1285.

Schneider, B. \& Seemüller, E. (1994). Presence of two sets of ribosomal genes in phytopathogenic mollicutes. Appl Environ Microbiol 60, 3409-3412.

Schneider, B., Ahrens, U., Kirkpatrick, B. C. \& Seemüller, E. (1993) Classification of plant-pathogenic mycoplasma-like organisms using restriction-site analysis of PCR-amplified 16S rDNA. J Gen Microbiol 139, 519-527.

Schneider, B., Gibb, K. S. \& Seemüller, E. (1997a). Sequence and RFLP analysis of the elongation factor Tu gene used in differentiation and classification of phytoplasmas. Microbiology 143, 3381-3389. 
Schneider, B., Marcone, C., Kampmann, M., Ragozzino, A., Lederer, W., Cousin, M.-T. \& Seemüller, E. (1997b). Characterization and classification of phytoplasmas from wild and cultivated plants by RFLP and sequence analysis of ribosomal DNA. Eur J Plant Pathol 103, 675-686.

Sears, B. B. \& Kirkpatrick, B. C. (1994). Unveiling the evolutionary relationships of plant-pathogenic mycoplasmalike organisms. ASM News 60, 307-312.

Sears, B. B., Lim, P.-O., Holland, N., Kirkpatrick, B. C. \& Klomparens, K. L. (1989). Isolation and characterization of DNA from a mycoplasmalike organism. Mol Plant-Microbe Interact 2, 175-180.

Seemüller, E. \& Schneider, B. (2004). 'Candidatus Phytoplasma mali', 'Candidatus Phytoplasma pyri' and 'Candidatus Phytoplasma prunorum', the causal agents of apple proliferation, pear decline and European stone fruit yellows, respectively. Int J Syst Evol Microbiol 54, 1217-1226.

Seemüller, E., Schneider, B., Mäurer, R. \& 8 other authors (1994). Phylogenetic classification of phytopathogenic mollicutes by sequence analysis of 16 S ribosomal DNA. Int J Syst Bacteriol 44, 440-446.

Seemüller, E., Marcone, C., Lauer, U., Ragozzino, A. \& Göschl, M. (1998). Current status of molecular classification of the phytoplasmas. J Plant Pathol 80, 3-26.

Seemüller, E., Garnier, M. \& Schneider, B. (2002). Mycoplasmas of plants and insects. In Molecular Biology and Pathology of Mycoplasmas, pp. 91-116. Edited by S. Razin \& R. Herrmann. London: Kluwer Academic/Plenum Publishers.

Shiomi, T. \& Sugiura, M. (1984). Grouping of mycoplasma-like organisms transmitted by the leafhopper vector, Macrosteles orientalis Virvaste, based on host range. Ann Phytopathol Soc Jpn 50, 149-157.

Smart, C. D., Sears, B. B. \& Kirkpatrick, B. C. (1994). Analysis of evolutionary relationships between MLOs and other members of the class Mollicutes based on 16/23S rRNA intergenic sequences. IOM Lett 3, 269-270.

Smart, C. D., Schneider, B., Blomquist, C. L., Guerra, L. J., Harrison, N. A., Ahrens, U., Lorenz, K. H., Seemüller, E. \& Kirkpatrick, B. C. (1996). Phytoplasma-specific PCR primers based on sequences of the 16S-23S rRNA spacer region. Appl Environ Microbiol 62, 2988-2993.

Stackebrandt, E. \& Goebel, B. M. (1994). Taxonomic note: a place for DNA-DNA reassociation and 16S rRNA sequence analysis in the present species definition in bacteriology. Int J Syst Bacteriol 44, 846-849.

Stackebrandt, E., Frederiksen, W., Garrity, G. M. \& 10 other authors (2002). Report of the ad hoc committee for the re-evaluation of the species definition in bacteriology. Int J Syst Evol Microbiol 52, 1043-1047.

Thompson, J. D., Gibson, T. J., Plewniak, F., Jeanmougin, F. \& Higgins, D. G. (1997). The CLUSTAL_X windows interface: flexible strategies for multiple sequence alignment aided by quality analysis tools. Nucleic Acids Res 25, 4876-4882.

Toth, K. F., Harrison, N. \& Sears, B. B. (1994). Phylogenetic relationships among members of the class Mollicutes deduced from rps3 gene sequences. Int J Syst Bacteriol 44, 119-124.

Tsai, J. H. (1979). Vector transmission of mycoplasmal agents of plant diseases. In The Mycoplasmas, vol. 3, pp. 265-307. Edited by R. E. Whitcomb \& J. G. Tully. San Diego, CANew York, NY: Academic Press.

Tymon, A. M., Jones, P. \& Harrison, N. A. (1998). Phylogenetic relationships of coconut phytoplasmas and the development of specific oligonucleotide PCR primers. Ann Appl Biol 132, 437-452.

Verdin, E., Salar, P., Danet, J.-L., Choueiri, E., Jreijiri, F., El Zammar, S., Gélie, B., Bové, J. M. \& Garnier, M. (2003). 'Candidatus Phytoplasma phoenicium', a novel phytoplasma associated with an emerging lethal disease of almond trees in Lebanon and Iran. Int J Syst Evol Microbiol 53, 833-838.

Vibio, M., Bertaccini, A., Lee, I.-M., Davis, R. E. \& Clark, M. F. (1996). Differentiation and classification of aster yellows and related European phytoplasmas. Phytopathol Mediterr 35, 33-42.

White, D. T., Blackall, L. L., Scott, P. T. \& Walsh, K. B. (1998). Phylogenetic positions of phytoplasmas associated with dieback, yellow crinkle and mosaic diseases of papaya, and their proposed inclusion in 'Candidatus Phytoplasma australiense' and a new taxon, 'Candidatus Phytoplasma australasia'. Int J Syst Bacteriol 48, 941-951.

Zreik, L., Carle, P., Bové, J. M. \& Garnier, M. (1995). Characterization of the mycoplasmalike organism associated with witches'-broom disease of lime and proposition of a "Candidatus" taxon for the organism, "Candidatus Phytoplasma aurantifolia". Int J Syst Bacteriol 45, 449-453. 